\title{
FUV Emission in Cool-Core Clusters
}

\section{J. B. R. Oonk ${ }^{1}$, W. Jaffe ${ }^{1}$, M. N. Bremer ${ }^{2}$, and N. Hatch ${ }^{1}$}

${ }^{1}$ Leiden Observatory, Leiden University, Leiden, The Netherlands

${ }^{2}$ H. H. Wills Physics Laboratory, University of Bristol, Bristol, UK

\begin{abstract}
Far ultraviolet (FUV) emission is observed in the central regions of cool-core clusters with the Hubble Space Telescope (HST). It is traced out to $20 \mathrm{kpc}$ from the nuclei of the brightest cluster galaxies and found to be distributed in clumps and filaments, as shown in Figure 1. The FUV emission matches the global structure of the ionized gas nebulae. If produced by stars, this emission can account for the ionization but not the temperature of the gas (Voit \& Donahue 1997; Oonk et al. in preparation).
\end{abstract}

Keywords. galaxies: galaxies: cooling flows, cD, clusters: general
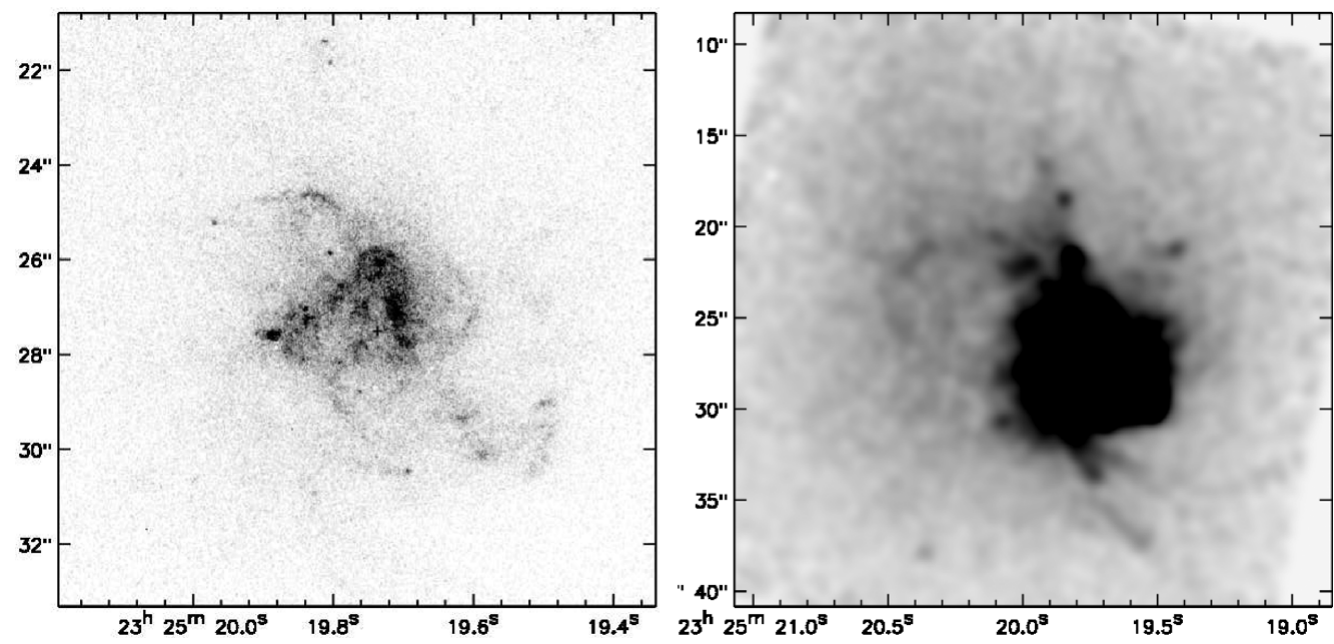

Figure 1. Far ultraviolet emission in the core of Abell $2597\left(1^{\prime \prime}=1.5 \mathrm{kpc}\right)$ as observed by HST. Left: FUV emission in the nuclear region at the intrinsic resolution of HST. Right: Extended FUV emission at a resolution of $1^{\prime \prime}$.

\section{Acknowledgements}

Observations for this project were accomplished with the NASA/ESA Hubble Space Telescope-ACS and the ESO Very Large Telescope-FORS.

\section{References}

Voit, G. M. \& Donahue, M. 1997, ApJ, 486, 242

Oonk, J. B. R., Hatch, N., Jaffe, W., \& Bremer, M. N., in preparation 